\title{
MRI-Derived Fetal Weight Estimation in the Midpregnancy Fetus: A Method Comparison Study
}

\author{
Jacqueline Matthew ${ }^{\mathrm{a}}$ Emily Skelton ${ }^{\mathrm{a}}$ Lisa Story ${ }^{\mathrm{a}, \mathrm{b}} \quad$ Alice Davidson $^{\mathrm{a}}$ \\ Caroline L. Knight ${ }^{a, b}$ Chandni Gupta ${ }^{c}$ Dharmintra Pasupathy ${ }^{d}$ \\ Mary Rutherford ${ }^{a, b}$
}

aSchool of Biomedical Engineering and Imaging Sciences and School of Life Course Sciences, Faculty of Life Sciences in Medicine, King's College London, London, UK; ' 'Guy's \& St. Thomas' NHS Foundation Trust, London, UK; 'North Tees and Hartlepool NHS Foundation Trust, London, UK; 'Westmead Clinical School, Faculty of Medicine and Health, University of Sydney, Sydney, NSW, Australia

\section{Keywords}

Biometry · Fetal weight $\cdot$ Magnetic resonance imaging · Second trimester · Ultrasound - Volumetry · Preterm .

Premature

\begin{abstract}
Objectives: The aim of this study was to compare the standard ultrasound (US) estimated fetal weight (EFW) and MRI volume-derived methods for the midtrimester fetus. Methods: Twenty-five paired US and MRI scans had the EFW calculated (gestational age [GA] range $=20-26$ weeks). The intra- and interobserver variability of each method was assessed ( 2 operators/modality). A small sub-analysis was performed on 5 fetuses who were delivered preterm (mean GA $29^{+3}$ weeks) and compared to the actual birthweight. Results: Two MRI volumetry EFW formulae under-measured compared to US by $-10.9 \%$ and $-14.5 \%$ in the midpregnancy fetus $(p<0.001)$ but had excellent intra- and interobserver agreement (intraclass correlation coefficient $=0.998$ and 0.993). In the preterm fetus, the mean relative difference (MRD) between the MRI volume-derived EFW (MRI-EFW) and actual expected birthweight (at the scan GA) was $-13.7 \%$
\end{abstract}

karger@karger.com www.karger.com/fdt

Karger $\stackrel{\text { ' }}{5}$

GOPEN ACCESS
C 2021 The Author(s).

Published by S. Karger AG, Basel

This article is licensed under the Creative Commons Attribution 4.0 International License (CC BY) (http://www.karger.com/Services/ OpenAccessLicense). Usage, derivative works and distribution are permitted provided that proper credit is given to the author and the original publisher.
$(-159.0 \mathrm{~g}, 95 \% \mathrm{Cl}:-341.7$ to $23.7 \mathrm{~g})$ and $-17.1 \%$ (-204.6 g, $95 \% \mathrm{Cl}:-380.4$ to $-28.8 \mathrm{~g}$ ), for the $2 \mathrm{MRI}$ formulae. The MRD was smaller for US at $5.3 \%(69.8 \mathrm{~g}, 95 \% \mathrm{Cl}:-34.3$ to 173.9$)$. Conclusions: MRI-EFW results should be interpreted with caution in midpregnancy. Despite excellent observer agreement with MRI volumetry, refinement of the EFW formula is needed in the second trimester, for the small and for the GA and preterm fetus to compensate for lower fetal densities.

(c) 2021 The Author(s).

Published by S. Karger AG, Basel

\section{Introduction}

Accurate estimated fetal weight (EFW) calculations are important to reliably screen for small for gestational age (SGA) fetuses and to detect and monitor fetal growth restriction (FGR) [1]. SGA typically refers to a fetus having an EFW or an abdominal circumference (AC) measurement of less than the 10th percentile for gestation or less than the 3rd percentile if severe [2]. FGR is not synonymous with SGA because some fetuses will be growthrestricted but achieve a normal birthweight; thus, FGR is considered a failure to achieve an "expected" growth po- 
tential $[3,4]$. Nonetheless, fetuses that are correctly identified as severely SGA are significantly associated with FGR and poorer outcomes.

Early delivery at extreme prematurity is often considered only when the EFW is above $500 \mathrm{~g}[2,5]$. Thus, an accurate EFW in the second and early third trimester is crucial to ensure timely and appropriate interventions. Yet, the ultrasound (US) biometry-derived EFW (USEFW) has significant random and inherent systematic variation (of up to $\pm 15 \%$ ) compared with birthweight, with errors more pronounced at the extremes of the normal range [6-9]. Recent studies suggest that a magnetic resonance imaging (MRI) whole fetal body volumebased EFW is far more reliable than the US-EFW at term, with errors compared to birthweight as low as 3\% [10-18]. In 1994, Baker et al. [19] published the first MRI volume-derived EFW (MRI-EFW) formula, and it is the most widely used in the literature. Kacem et al. [14] proposed an alternative to the Baker formula and considered varying fetal density across the gestational age (GA) range. There is less evidence about the reliability of the MRI-EFW at a GA remote from term, when adverse health outcomes related to FGR can be more severe.

US operator training and audit is unlikely to improve measurement errors within US-EFW calculations significantly; thus, development of alternative methods is required [6]. 3D-US has shown some promise however has not been routinely adopted in the clinical setting because it is yet to be evidenced as being more reliable than 2D-US biometric methods [20-22]. The primary aim of this study was to compare the agreement and reliability of US and MRI to measure the EFW in the second trimester and a secondary aim to assess the feasibility of the MRI-EFW in cases of extreme prematurity.

\section{Materials and Methods}

Healthy pregnant participants were prospectively recruited between November 2015 and April 2016 as part of the ethically approved intelligent fetal imaging and diagnosis (iFIND) project (ethics number: 14/LO/1806). Inclusion criteria were normal 20week US anatomy scan, paired US and MRI data within 3 days of each other, and GA between 20 and 28 weeks.

For the secondary aim, participants at high-risk of preterm birth (PTB) were prospectively recruited between December 2015 and October 2017, for the "MRI quantification of fetal growth and development study" and as part of a subgroup described in 2020 by Story et al. [23] (ethics number 07/H0707/105). Inclusion criteria were GA 20-32 weeks, high-risk of PTB (i.e., asymptomatic women with either a history of previous $\mathrm{PTB}$, late miscarriage $[>16$ weeks], or cervical surgery with a $>50 \%$ risk of PTB in the next 2 weeks [calculated using a fetal fibronectin and cervical length algorithm]) [24, 25], and paired US and MRI data both within 10 days of delivery. Exclusion criteria for both control and high-risk PTB fetuses were known structural or chromosomal abnormalities, multiple pregnancies, inability to give informed consent, pregnancy complications such as pre-eclampsia or gestational diabetes, and contraindications to MRI such as claustrophobia or a recently sited metallic implant.

\section{Method Comparison of the EFW in the Second Trimester}

The design for the primary aim was a prospective, blinded, within-subject paired method comparison, observer agreement, and reliability study at a single center. A Philips EpiQ US system (Philips Healthcare, Best, Netherlands) with a 6-1-MHz matrix probe was used to scan all control participants by 1 of 2 observers (J.M./C.K.) in a dedicated research US clinic. 2D-US anatomical image planes including the transventricular view of the fetal head for the head circumference $(\mathrm{HC})$, transverse abdomen view for the $\mathrm{AC}$, and long axis of the femur for the femur length (FL) measurement were identified during each examination and stored. Image plane selection criteria were obtained from the NHS Fetal Anomaly Screening Programme guidelines [26]. An image database containing anonymised US DICOM images was compiled using the Osirix image review software for offline measurement (version 7.5, Geneva, Switzerland). US databases were duplicated and randomised using a computer-generated randomiser before being reviewed offline for inter- and intraobserver variability by the 2 fetal imaging experts, blinded to previous imaging results and clinical history (including GA) as previously described [27]. Both US-observers used the first US database to independently measure 2DUS fetal biometry for interobserver measurements, and then USobserver 1 repeated the measures after a 6-week interval to generate intraobserver measurements.

The US-EFW was then calculated from the $\mathrm{HC}, \mathrm{AC}$, and $\mathrm{FL}$ biometry using the 3-parameter 1985 Hadlock formula [28]:

US - EFW $(\mathrm{g})=10^{(1.326-0.00326 \times \mathrm{AC} \times \mathrm{FL}+0.0107 \times \mathrm{HC}+0.0438 \times \mathrm{AC}+0.158 \times \mathrm{FL})^{4}}$

The fetal MRI scan was performed using a Philips Ingenia 1.5 T MRI system (Philips Healthcare, Best, Netherlands). The mother was placed in a left lateral tilt, and no sedation was used for the examination. A sagittal plane orientated to the fetus was planned to acquire a balanced turbo field echo sequence which provided optimal image contrast resolution, coverage of the region of interest, and speed of acquisition (field of view $=420 \times 420 \mathrm{~mm}$; matrix $=288 \times 288$; repetition time $=4 \mathrm{~ms}$; echo time $=1.98 \mathrm{~ms}$; slices $=$ 91; slice thickness $=5 \mathrm{~mm}$; slice overlap $=2.5 \mathrm{~mm}$; noise signal averages $=1 /$ SENSE $=$ yes [2]; flip angle $=90^{\circ}$; acquisition time $=$ $1 \min 25 \mathrm{~s}$ ). MRI data from all the subjects were anonymized and randomized before being distributed to the fetal imaging experts (J.M./A.D.) for independent volume segmentation, blinded to USEFW results and clinical history. Both MRI-observers calculated MRI whole fetal body volumes with a semiautomatic thresholding technique, and then performed manual slice-by-slice editing of the segmentation using open-source software, ITK-Snap (version 2.2.0), ${ }^{33}$ for interobserver measurements (see Fig. 1). All fetal body tissues were included in the segmentation even if some fetal tissues appeared misaligned between slices. Observer 1 (J.M.) performed repeated measures after a 6-week interval to generate intraobserver measurements. 
Table 1. Operator experience

Role

US-observer 1

US-observer 2

Reporting obstetric sonographer

3

MRI-observer 1

Fetal medicine consultant

Reporting obstetric Sonographer/MRI radiographer 13

MRI-observer 2

Fetal MRI data analyst/researcher

Preterm Birth MRI observer

Fetal medicine sub-specialty trainee/clinical academic

19]:

The MRI-EFW was calculated using the 2 formulae below [14,

- Volume $\operatorname{EFW}_{\text {Baker }}(\mathrm{kg})=1.031 \times$ fetal body volume $(\mathrm{L})+0.12$ (kg).

- Volume $\operatorname{EFW}_{\text {Kacem }}(\mathrm{kg})=0.989 \times$ fetal body volume $(\mathrm{L})+0.147$ (kg).

All observers were provided with face-to-face training and given written guidance notes prior to the review explaining the required measurements, segmentation technique, and optimal viewing conditions for the review.

\section{Data Analysis}

Data were recorded on an Excel spreadsheet (version 15.0, Microsoft Corp, Redmond, WA, USA) and analyzed using SPSS (version 26, SPSS Inc., Chicago, IL, USA). Statistical analysis was performed as per recommended guidelines to avoid study reporting variation [29-33]. For the primary aim, a power calculation determined that a sample size of 20 was required to give a power of $80 \%$ for a type 1 error of $5 \%$ to detect an effect size of $13.0 \mathrm{~g}$ difference (assuming a standard deviation of $104 \mathrm{~g}$ based on previous studies) [27, 34].

Normality testing was performed to ensure assumptions were met for statistical analysis. To assess the systematic differences between modalities, the mean difference in measurement from $2 \mathrm{ob}-$ servers per modality was compared with a 2 -tailed paired $t$ test. The average measures intraclass correlation coefficient (ICC) was used to test the intra- and interobserver agreement, with $95 \%$ confidence intervals. Predefined cutoff limits for the ICC were used: $>0.99$, very good; $0.95-0.99$, good; $0.90-0.95$, moderate; $0.70-0.90$, poor; and $<0.70$, very poor [32]. Bland-Altman plots were used to graphically assess the mean difference in observations and their limits of agreement (LoA), and a linear regression coefficient was used to determine if there was a statistically significant proportional bias in the error as the fetal size increased. Finally, the proportion of cases which fell outside of 2 specified error thresholds (5\% and 10\%) was calculated.

\section{Preterm MRI-EFW Feasibility}

Using the same MRI segmentation method, preterm fetuses meeting the inclusion criteria had the MRI-EFW calculated by a single observer (L.S.). The MRI-EFW of PTB cases were compared with the most recent clinical US-EFW and the actual birthweight $(\mathrm{ABW})$ at delivery. Additionally, weight centiles were calculated from newborn and fetal population-based growth chart calculators $[35,36]$. The ABW centiles were used to compute the expected EFW for each case and thus correct for any time interval between GA at delivery and GA at the time of the scan. The absolute and percentage differences between the expected and actual EFW were calculated for MRI and US.

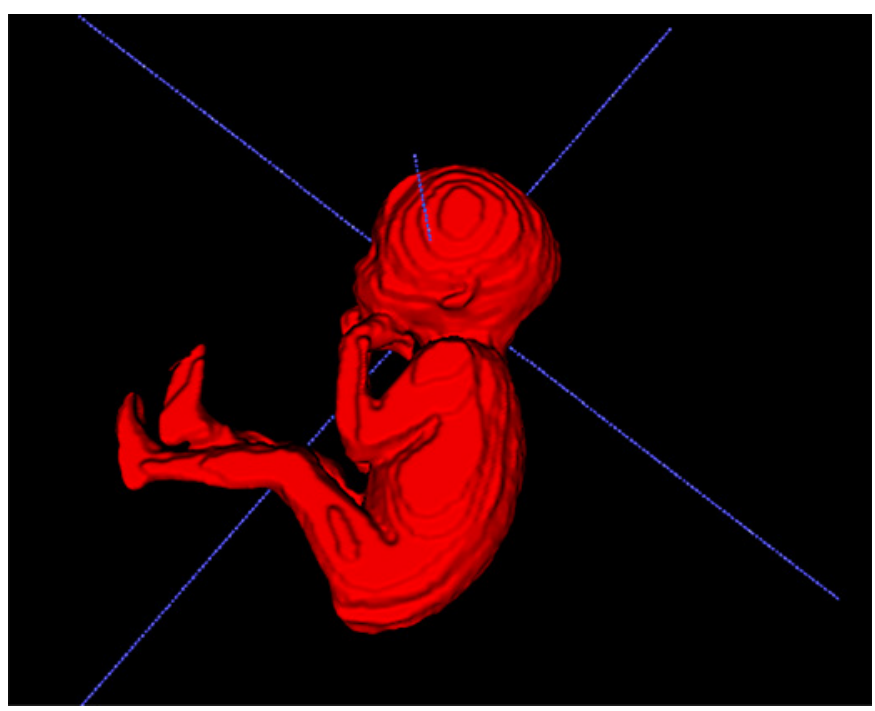

Fig. 1. Semiautomatic segmentation of MRI whole fetal body volume.

\section{Results}

\section{Observer and Subject Demographics}

Twenty-five control cases met the inclusion criteria for the study's primary aim. The mean maternal age at the time of the scan was 32.5 years (range 26-39). The mean maternal BMI was $26.3 \mathrm{~kg} / \mathrm{m}^{3}$ (range $22.2-38.4 \mathrm{~kg} / \mathrm{m}^{3}$ ). The mean gestational age at time of consent (i.e., the first scan) was $23^{+4}$ weeks (range $20^{+2}-25^{+5}$ weeks). Seventeen participants $(68 \%)$ underwent US and MRI on the same day, and 7 (32\%) had the MRI scan exactly 3 days after US. Five cases did not have outcome data available as they delivered at a different center and were lost to follow-up. Of the 20 cases with outcome data, there was 1 preterm delivery at $31^{+3}$ weeks gestation which was included as a control because MRI and US examinations occurred within a short time interval. Including the preterm case, the median gestational age at delivery was $39^{+4}$ (range $31^{+3}-42^{+0}$ weeks), and the median birthweight was 
Table 2. Mean US-EFW (Hadlock formula) and MRI-EFW (Baker and Kacem formulae) for 25 midpregnancy fetuses in grams (SD)

\begin{tabular}{lllll}
\hline & Observer 1 & Observer 2 & $\begin{array}{l}\text { Observer 1 } \\
\text { (repeated measure) }\end{array}$ & $\begin{array}{l}\text { Mean } \\
\text { (observer 1 and observer 2) }\end{array}$ \\
\hline Mean US-EFW, Hadlock & $653.3(106.4)$ & $641.0(103.6)$ & $682.6(113.7)$ & $647.1(104.2)$ \\
Mean MRI-EFW, Baker & $579.5(99.3)$ & $573.5(99.6)$ & $579.7(99.8)$ & $576.5(99.1)$ \\
Mean MRI-EFW, Kacem & $556.1(95.8)$ & $550.1(95.5)$ & $555.9(95.3)$ & $553.0(95.1)$ \\
\hline
\end{tabular}

SD, standard deviation; US-EFW, ultrasound biometry-derived estimated fetal weight; MRI-EFW, MRI volume-derived estimated fetal weight.

Fig. 2. Boxplot of the EFW for health controls using US and MRI (Baker and Kacem) methods. EFW, estimated fetal weight; US, ultrasound; US-EFW, ultrasound biometry-derived estimated fetal weight; MRIEFW, MRI volume-derived estimated fetal weight.

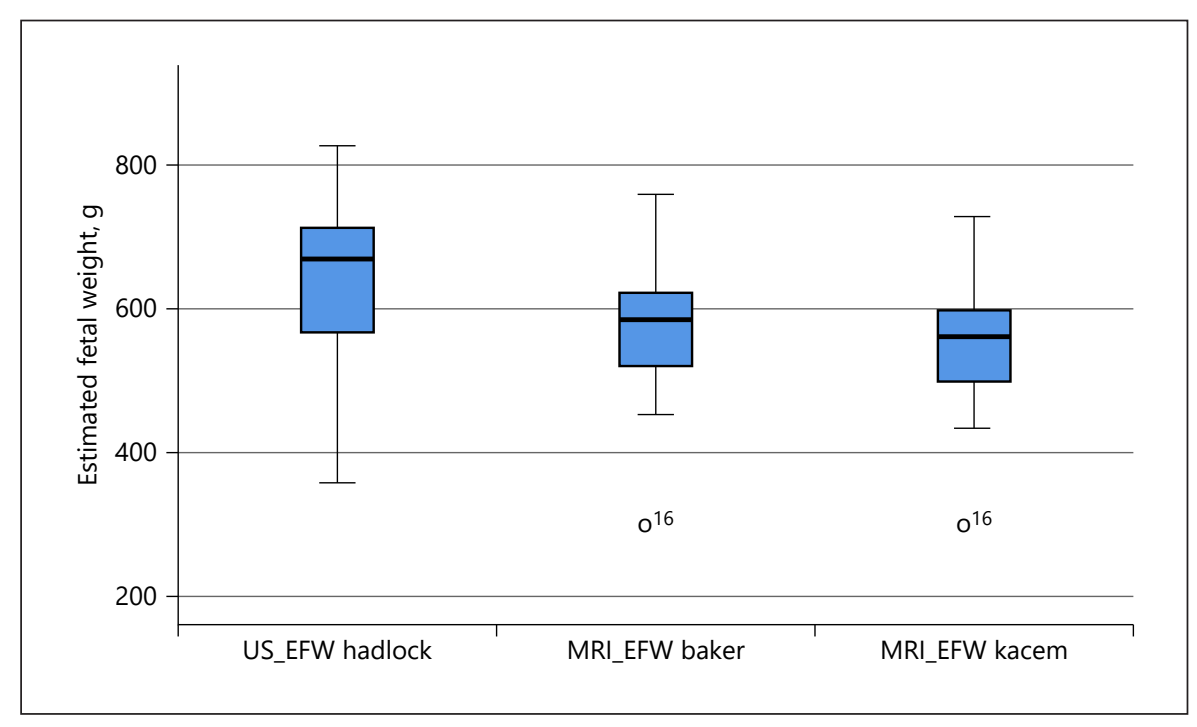

$3,240.0 \mathrm{~g}$ (range 1,850-4,480 g). For the method comparison, agreement, and reliability assessment, a total of 225 US images from 25 unique control subjects were measured by 2 US observers. This consisted of 75 images per database review, with US-observer 1 repeating the measures. A total of $75 \mathrm{MRI}$-observations from 25 unique MRI balanced turbo field echo sequences had a volume segmentation performed by 2 MRI observers in the same 25 control subjects, with MRI-observer 1 repeating the measures (see Table 1 for observer experience). For the secondary aim of the study, there were 5 high-risk PTB cases meeting the inclusion criteria for the feasibility aim of this study. The mean gestational age at the time of MRI was $29^{+3}$ weeks (range $25^{+6}-31^{+3}$ weeks) and at US was $29^{+1}$ weeks (range $24^{+6}-31^{+3}$ weeks). The mean gestational age at delivery was $29^{+6}$ weeks (range $26^{+1}-31^{+6}$ weeks), and all were delivered within 9 days (median 5 days) of the US examination and 5 days (median 2 days) of the MRI scan. The mean ABW was 1,310 g, range 770-1,690 g. The 5 PTB cases had a clinical US examination per- formed by 3 different operators, and the MRI segmentation was performed by a single observer experienced in this method.

\section{Descriptive Statistics}

The MRI calculation using both formulae produce a smaller EFW in the same group of fetuses than the USEFW, and the standard deviation is smaller for MRI than US, with the Kacem formula resulting in the smallest EFW and the least variation (see Fig. 2; Table 2). These differences demonstrate a similar pattern for the cases that delivered preterm (Fig. 3; Table 3).

\section{Differences between US and MRI for EFW (Healthy} Controls)

Q-Q plots for normality testing demonstrated a linear relationship for EFW parameters, and a nonsignificant $(<0.05)$ Shapiro-Wilk result was calculated. When compared to US-EFW, both MRI formulae consistently and significantly under-measure EFW. For the Baker method, 
Table 3. High-risk preterm birth results for ABW and expected and actual EFW for US and MRI

\begin{tabular}{|c|c|c|c|c|c|c|c|c|c|c|}
\hline Case & GA delivery & $\begin{array}{l}\text { US to delivery } \\
\text { interval, days }\end{array}$ & $\begin{array}{l}\text { MRI to delivery } \\
\text { interval, days }\end{array}$ & $\begin{array}{l}\mathrm{ABW} \\
\mathrm{g}\end{array}$ & $\begin{array}{l}\text { ABW } \\
\text { centile }^{\$}\end{array}$ & $\begin{array}{l}\text { Expected } \\
\text { US-EFW, } \mathrm{g}^{*}\end{array}$ & $\begin{array}{l}\text { Actual } \\
\text { US-EFW, g }\end{array}$ & $\begin{array}{l}\text { Expected } \\
\text { MRI-EFW, g* }\end{array}$ & $\begin{array}{l}\text { Actual MRI- } \\
\text { EFW }_{\text {Baker }} \mathrm{g}\end{array}$ & $\begin{array}{l}\text { Actual MRI- } \\
\text { EFW }_{\text {Kacem }} \mathrm{g}\end{array}$ \\
\hline$A$ & $26+1$ & 9 & 2 & 770 & $23 r d$ & 664 & 700 & 745 & 534 & 513 \\
\hline$B$ & $31+5$ & 2 & 2 & 1,690 & 55 th & 1,637 & 1,827 & 1,637 & 1,518 & 1,456 \\
\hline C & $31+6$ & 6 & 5 & 1,400 & 11 th & 1,284 & 1,389 & 1,302 & 1,136 & 1,090 \\
\hline D & $31+0$ & 5 & 3 & 1,540 & 50 th & 1,419 & 1,476 & 1,466 & 1,306 & 1,253 \\
\hline $\mathrm{E}$ & $28+1$ & 5 & 0 & 1,150 & 55 th & 1,052 & 1,144 & 1,150 & 1,150 & 1,104 \\
\hline Mean & $29+3$ & 5 & 2 & 1,310 & 39th & 1,211 & 1,307 & 1,260 & 1,129 & 1,083 \\
\hline SD & (Range $26+1$ to $31+6$ ) & 2.2 & 1.6 & 323.3 & 20.5 & 333.0 & 374.4 & 304.5 & 327.8 & 314.1 \\
\hline
\end{tabular}

ABW, actual birthweight; EFW, estimated fetal weight; US-EFW, ultrasound biometry-derived estimated fetal weight; MRI-EFW, MRI volume-derived es-

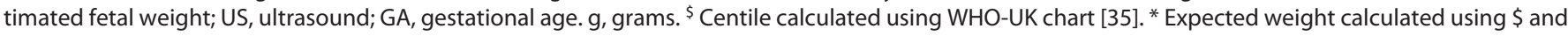
Intergrowth fetal charts [36].

Table 4. Paired $t$ test of the differences between the mean US-EFW and MRI-EFW (Baker and Kacem)

\begin{tabular}{|c|c|c|c|c|c|}
\hline US versus MRI Baker EFW & 647.1 & 576.5 & $70.7(50.1-91.2)$ & -10.9 & $<0.001$ \\
\hline \multirow[t]{2}{*}{ US versus MRI Kacem EFW } & 647.1 & 553.0 & $94.1(73.8-114.5)$ & -14.5 & $<0.001$ \\
\hline & $\begin{array}{l}\text { Mean MRI } \\
\text { Baker EFW, g }\end{array}$ & $\begin{array}{l}\text { Mean MRI Ka- } \\
\text { cem EFW, g }\end{array}$ & $\begin{array}{l}\text { Absolute mean paired difference, } \\
\text { g (95\% confidence interval) }\end{array}$ & $\begin{array}{l}\text { Relative mean paired } \\
\text { difference, } \%\end{array}$ & $p$ value \\
\hline MRI Baker versus MRI Kacem EFW & 576.5 & 553.0 & $23.5(21.8-25.1)$ & $-4.1 \%$ & $<0.001$ \\
\hline
\end{tabular}

US-EFW, ultrasound biometry-derived estimated fetal weight; MRI-EFW, MRI volume-derived estimated fetal weight; US, ultrasound.

Fig. 3. Boxplot of the EFW using US and MRI (Baker and Kacem) methods and the $\mathrm{ABW}$ for pregnancies resulting in preterm births. EFW, estimated fetal weight; US, ultrasound; ABW, actual birthweight; USEFW, ultrasound biometry-derived estimated fetal weight; MRI-EFW, MRI volume-derived estimated fetal weight.

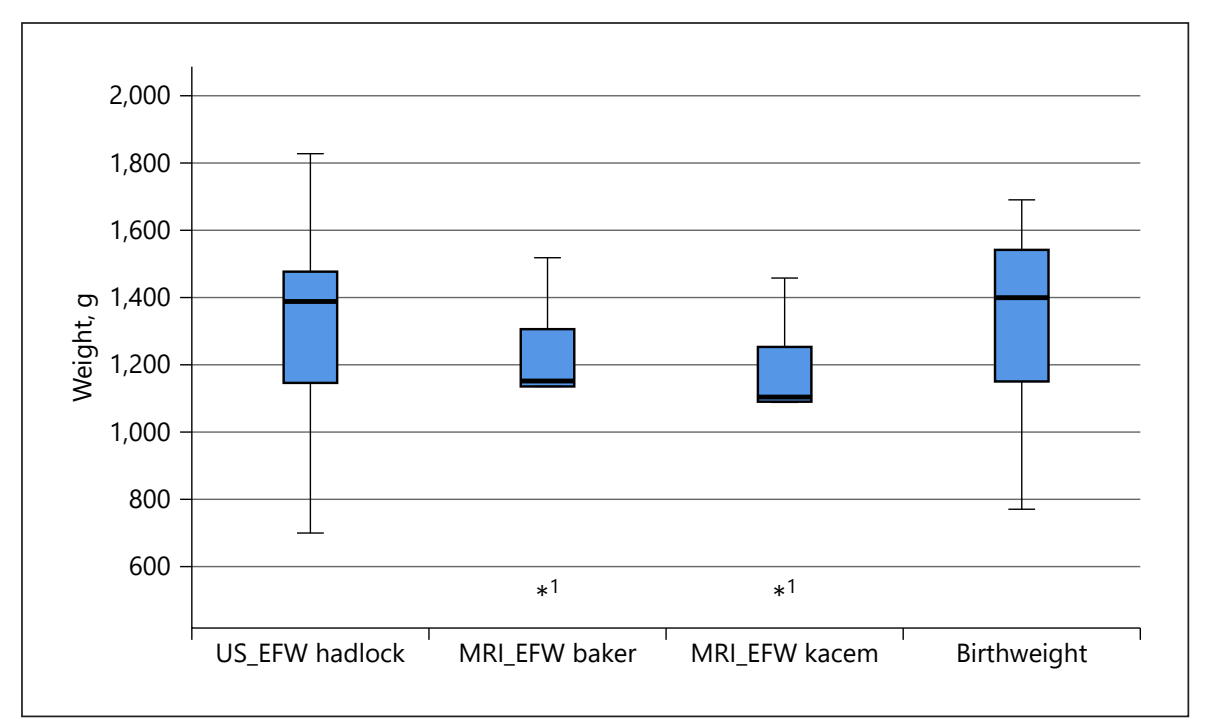

the mean percentage error was $-10.9 \%$ (70.7 $\mathrm{g}$ ), and for the Kacem method, the mean percentage error was $-14.5 \%$ (94.1 g). A significant difference between the 2
MRI-EFW methods was also demonstrated with a percentage error of $-4.1 \%$ (23.5 g). All paired $t$ test $p$ values were significant and $<0.001$ (see Table 4 ). 
Table 5. Intra- and interobserver agreement, ICC (95\% confidence intervals), linear regression $p$ value for proportional bias

\begin{tabular}{|c|c|c|}
\hline & US-EFW & MRI-EFW \\
\hline Intraobserver (within observer) & $0.972, \operatorname{good}(0.558-0.993) p=0.268$ & 0.998, excellent $(0.989-0.998) p=0.874$ \\
\hline Interobserver (between observers) & $0.984, \operatorname{good}(0.956-0.993) p=0.254$ & 0.993, excellent $(0.984-0.997) p=0.659$ \\
\hline
\end{tabular}

ICC, intraclass correlation coefficient; US-EFW, ultrasound biometry-derived estimated fetal weight; MRI-EFW, MRI volume-derived estimated fetal weight.

Table 6. (a) Absolute and relative inter- and intraobserver mean difference, with single measures $t$ test and (b) absolute and relative interand intraobserver mean paired difference between US and MRI, with a paired $t$ test

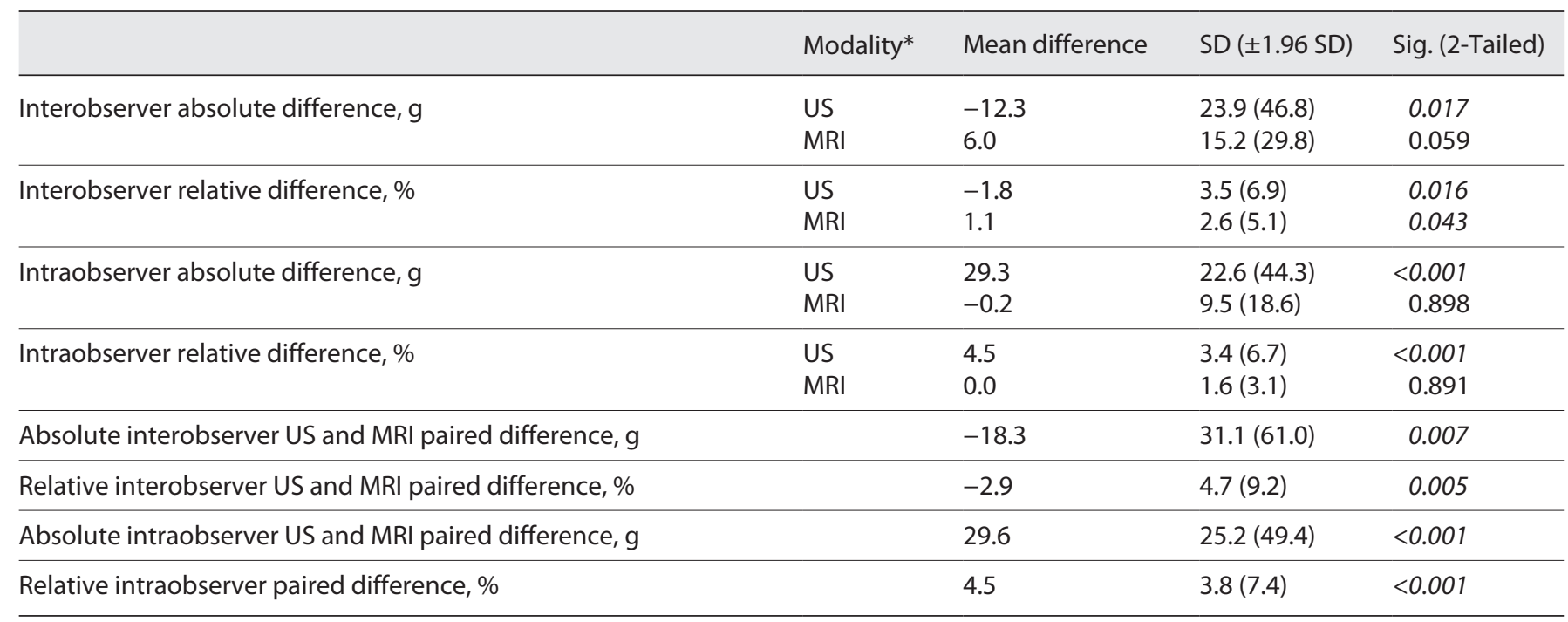

g, grams; \%, percentage; SD, standard deviation; sig., significance, presented as a $p$ value (italics $=p$ value $<0.05$ ). US, ultrasound; EFW, estimated fetal weight. ${ }^{*}$ As both MRI formulae used the same fetal volume measurement, their relative variation (random error) will be the same; therefore, the Kacem generated EFW is not presented.

Inter- and Intraobserver Agreement

Excellent ICC scores were generated for intraobserver (0.998) and interobserver (0.993) MRI-EFW agreements. In comparison, the ICC score for the US-EFW was good for both intraobserver (0.972) and interobserver (0.984) agreements (see Table 5). The 95\% confidence intervals overlap between US and MRI methods for both intra- and interobserver measurements, suggesting there is no significant difference in observer agreement between the modalities. The linear regression performed to assess proportional bias gave a statistical $p$ value of $>0.05$ for every ICC result. This suggests that the agreement of US and MRI is independent of the overall size of the measurement taken at this GA range.
Intra- and Interobserver Bland-Altman Plots of the US-EFW and MRI-EFW

The intraobserver mean relative (percentage) error of the MRI-EFW is lower than that of the US-EFW ( 0 and $4.5 \%$, respectively) which indicates excellent MRI agreement, with US discordance being equivalent to $29.3 \mathrm{~g}$ which is statistically significant (see Table 6a). The interobserver relative mean percentage errors for the MRIEFW and US-EFW are equally small ( -1.8 and $1.1 \%$, respectively), which represents an US mean absolute difference of $-12.3 \mathrm{~g}$ to MRI's $6.0 \mathrm{~g}$, with the US difference reaching statistical significance. The $t$ test for the direct comparison of US and MRI mean paired differences reached statistical significance, suggesting the observer variation between the modalities when measuring the EFW is real (see Table 6b). 


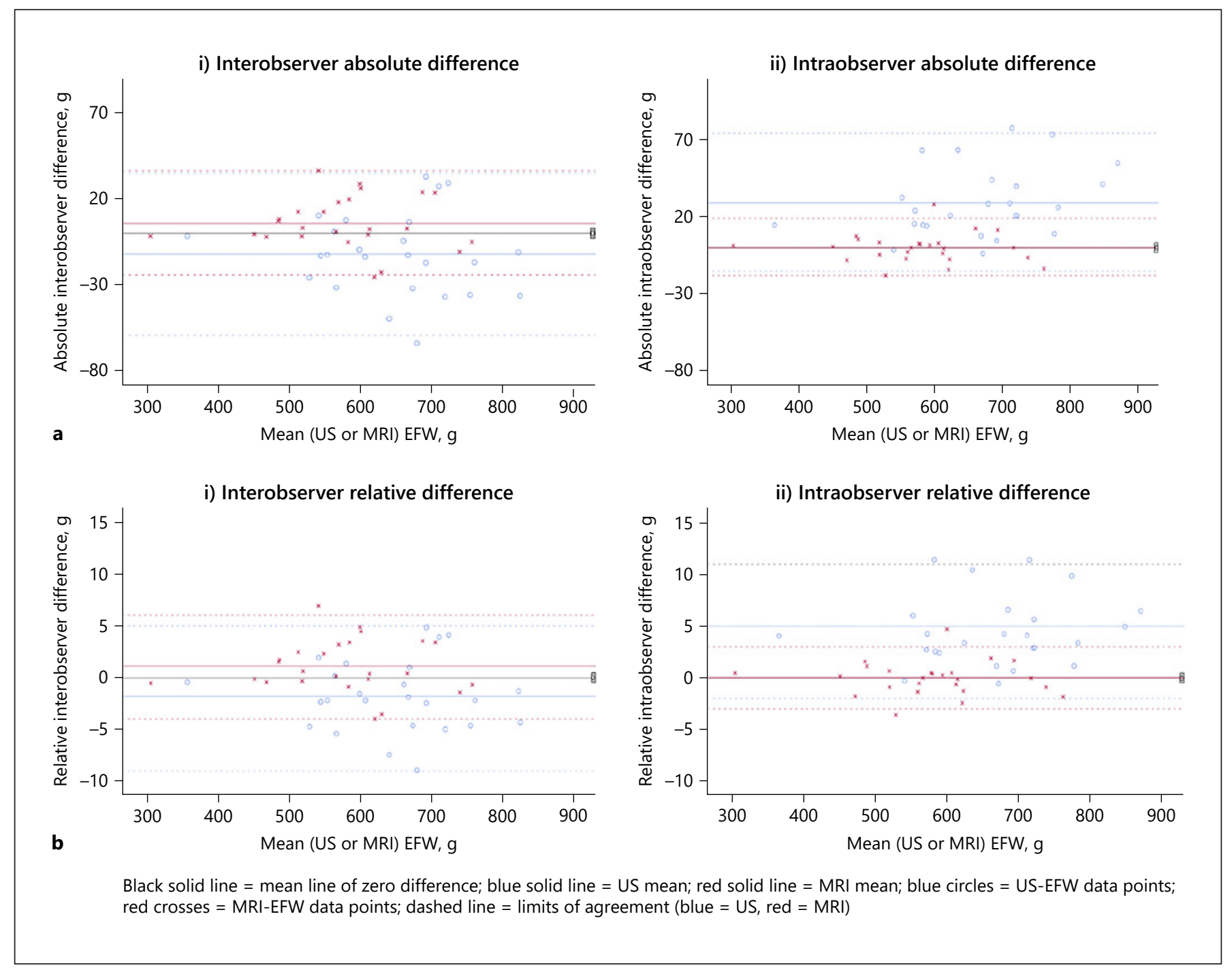

Fig. 4. Bland-Altman plots of the absolute (a) and relative (b) differences for interobserver (i) and intraobserver (ii) EFW measures. EFW, estimated fetal weight; LoA, limits of agreement.

Bland-Altman plots graphically represent the absolute and relative mean difference and the LoA, that is, the variation in $95 \%$ of the dataset (or \pm 1.96 SD from the mean) for each US-EFW and MRI-EFW datapoint (see Fig. 4). US-EFW LoA is wider than that for the MRIEFW for both intra- and interobservations, suggesting MRI is more precise. For interobserver MRI-EFW measures, $95 \%$ of the cases are within $\pm 29.8 \mathrm{~g}$, and for USEFW, they are within $\pm 44.8 \mathrm{~g}$ (see Table $6 \mathrm{a}$ ). EFW datapoints for both MRI and US appear randomly spread on the charts, which suggests no proportional bias and confirms the findings of the linear regression statistics (Table 5).

\section{Absolute Error Thresholds}

Threshold values for an arbitrary, but clinically relevant, cut off in variability (random error) for the EFW of $10 \%$ and then $5 \%$ assess the proportion of cases which fell outside these ranges compared to the mean of the intraobserver repeated measures which are usually the smallest in error (Table 7). For the US-EFW, there were 2 out of 25 cases (8\%) for intraobserver calculations, but no interobserver cases that fell outside of the $10 \%$ error threshold. However, there were $8 / 25$ cases (32\%) and 5/25 (20\%) for intra- and interobserver calculations, respectively, that fell outside of the 5\% threshold. For the MRI-EFW, there was only 1 case that had an error of $>5 \%$. There were 
Table 7. Proportion of US-EFW and MRI-EFW cases falling outside of $10 \%$ and $5 \%$ arbitrary error threshold

\begin{tabular}{|c|c|c|c|c|c|}
\hline \multirow{2}{*}{$\begin{array}{l}\text { Arbitrary cutoff } \\
\text { percentage }\end{array}$} & \multirow{2}{*}{$\begin{array}{l}\text { Threshold value, } \\
\text { grams, g }\end{array}$} & \multicolumn{2}{|l|}{ Intraobserver } & \multicolumn{2}{|l|}{ Interobserver } \\
\hline & & US-EFW n (\%) & MRI-EFW*, $n(\%)$ & US-EFW, $n(\%)$ & MRI-EFW*, $n(\%)$ \\
\hline US-EFW 10\% & 66.8 & $2(8)$ & & $0(0)$ & \\
\hline US-EFW 5\% & 33.4 & $8(32)$ & & $5(20)$ & \\
\hline MRI-EFW 10\% & 58.0 & & $0(0)$ & & $0(0)$ \\
\hline MRI-EFW 5\% & 29.0 & & $0(0)$ & & $1(4)$ \\
\hline
\end{tabular}

EFW, estimated fetal weight; US-EFW, ultrasound biometry-derived estimated fetal weight; MRI-EFW, MRI volume-derived estimated fetal weight. * As both MRI formulae used the same fetal volume measurement, their random error will be the same; therefore, the Kacem generated EFW is not presented.

Table 8. High-risk PTB absolute and relative differences between expected and actual EFW for US and MRI

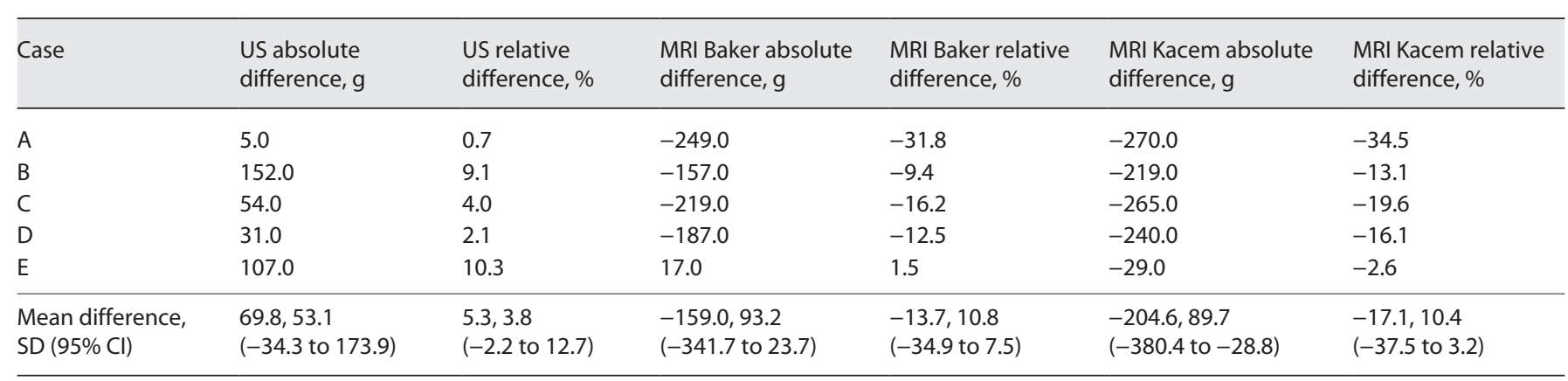

EFW, estimated fetal weight; PTB, preterm birth; US, ultrasound.

no other cases falling outside the error threshold for the MRI-EFW, confirming the precision of MRI over US.

\section{Preterm Sub-Analysis}

For the 5 preterm fetuses, all were delivered within 5 days of the MRI examination, and when the expected estimated weight was calculated using the ABW centiles and thus controlling for GA, there was a clinically significant mean difference between the actual and expected MRIEFW of $-13.7 \% /-159.0 \mathrm{~g}$ (Baker) and $-17.1 \% /-204.6 \mathrm{~g}$ (Kacem) (see Tables 3, 8). In contrast to the MRI-EFW, the US-EFW overmeasured birthweight compared with the expected EFW by a mean of 5.3\%/69.8 g. In 1 case, the MRI-EFW demonstrated a smaller relative difference than US, $1.5 \%-10.1 \%$, respectively (case E). However, 4 of 5 MRI cases had a relative difference from the expected EFW of $>5 \%$. For the US 2 cases, cases $\mathrm{B}$ and $\mathrm{E}$ had a relative difference of $>5 \%$. When comparing the weight centiles, corrected for GA at the time of scan, US appeared to overmeasure consistently compare to the ABW centile and MRI appeared to under-measure consistently, except in case E, where the MRI estimate was accurate (see Fig. 5).

\section{Discussion}

Our study showed higher observer reliability for MRIEFW calculations than for US-EFW calculations, suggesting better reproducibility, repeatability, and precision of the MRI method in the second trimester. Yet, caution must be exercised if using this technique for the EFW in small fetuses as the MRI calculation showed systematic measurement differences compared to US on which fetal growth trajectory charts are based.

A recent literature search revealed no studies focusing on volumetric MRI-EFW for mid-second trimester fetuses using either the Baker or the Kacem formula and then comparing the results to US and/or birthweight. However, a few studies have looked at 2D-MRI biometry to estimate fetal weight in the second trimester with limited success $[27,37]$. Our results contrast with recent findings by Kadji et al. [10] who assessed observer variability in EFW calculation for MRI and US in full-term fetuses. In their study, the mean relative error in the EFW difference for MRI and US was $0.9 \%$ and $-0.8 \%$, respectively, for intraobserver measures $(0.0 \%$ and $-4.5 \%$ in our study) 
Fig. 5. Actual US and MRI-EFW centiles, corrected for GA at the time of scan, compared to the birthweight centile. EFW, estimated fetal weight; US, ultrasound; GA, gestational age.

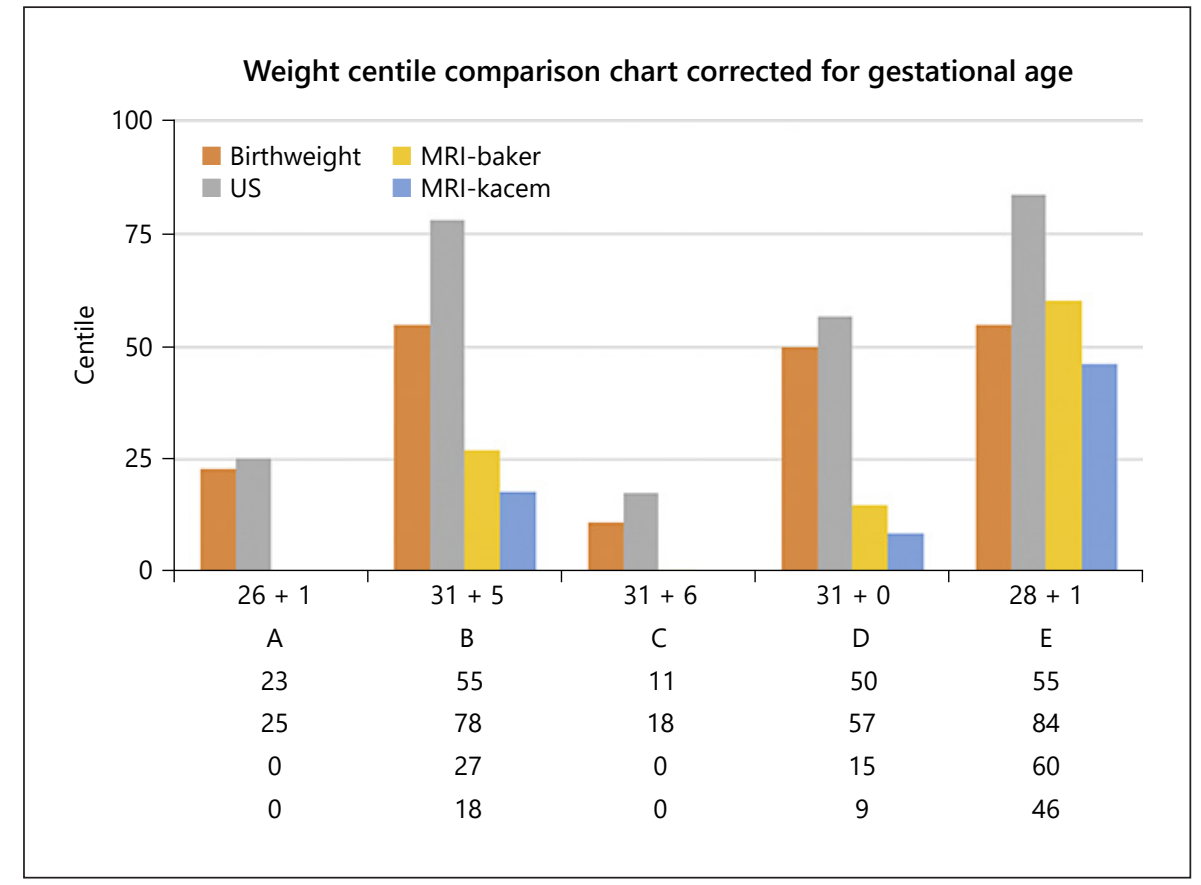

and $0.6 \%$ and $0.5 \%$ for interobserver measurements $(1.1 \%$ and $-1.8 \%$ in our study). Effectively, Kadji et al. [10] suggest excellent agreement for both modalities; however, our study agreement suggests excellent MRI agreement but with US being slightly less precise within and between observers. Other studies report that a significant proportion of US random errors (between 58 and 80\%) is incurred through observer variations in caliper placement, and for these cases, training and quality audit will help to some degree but not entirely [14, 38-40].

Kadji et al. [41] found a random error of $\pm 1.9 \%$ and $8.8 \%$ in MRI and US, respectively, for intraobserver measures $( \pm 1.6 \%$ and $3.4 \%$ in our study) and $\pm 2.8 \%$ and $11.2 \%$ for interobserver measurements $( \pm 2.6 \%$ and $3.5 \%$ in our study). The larger US errors reported by Kadji et al. [41] are likely to reflect increased proportional bias, observed when random errors increase as US measurements become larger at a later GA (in this case, term), a phenomenon described by obstetric US-observer variation studies [38]. In our sample (second trimester), this high proportional variation is not seen with US due to smaller fetal sizes. Nonetheless, the MRI-EFW is less susceptible to higher proportional variation likely because of the use of one volumetric parameter with well-defined landmarks rather than linear measurements subject to the US caliper-placement error. In our study, as in the Kadji study, regression analyses found no statistically significant difference in EFW variation as a result of increasing
GA; however, these findings are based on narrow GA ranges under observation [29].

The PREMACRO study [41] found that for term fetuses, the mean relative errors of MRI were between 2.6$3.7 \%$ and $6.3-11.4 \%$ for the US-EFW, compared to birthweight when calculated $<1$ week of delivery. Other studies also found small relative differences (between 3 and 4\%) for MRI volume weight estimates using the Baker formula when compared to the weight at term $[14,15,19,42]$. Our preterm sub-analysis compared the actual MRI-EFW to the expected EFW based on the ABW centile, and the mean relative error was larger and clinically significant for the MRI-EFW than the US-EFW ( -14 to $-17 \%$ and $5 \%$, respectively). While larger studies would be required to investigate these contrasting findings further, it is an important study because it suggests that while the MRI-EFW may have less observer variation, the MRI-EFW calculation demonstrates systematic under-measuring of the fetal weight at GAs remote from term when compared to the US estimated and ABW, whereas the literature suggests very good MRI performance $>37$ weeks.

The Kacem study found the proportion of cases that fell outside the $\pm 10 \%$ random error threshold was $26.6 \%$ for US cases and $1.1 \%$ for MRI, when the EFW was compared to birthweight (20\% and $4 \%$ for US and MRI interobserver variation with stricter $5 \%$ threshold in our study) [14]. In 2003, Zarestsky et al. [15] found $15 \%$ of US and $5 \%$ of MRI cases fell outside the ABW $\pm 10 \%$ thresh- 
old, and when using a $\pm 5 \%$ error threshold, $73 \%$ of US cases and $49 \%$ of MRI cases fell outside the threshold [15].

The segmentation process in our study used a manual and semiautomated method; however, more recently automated planimetry techniques have been described to produce the MRI fetal body volume [43]. Although small errors were seen for the MRI-EFW, the segmentation technique and choice of acquisition sequence may have an impact on the differences between studies. In addition, varying fetal tissue density (fat, bone, and muscle) at different gestations may be responsible for the MRI-EFW formula not performing well remotely from term [16]. Kacem used a linear regression model to attempt to address this issue, modifying the original Baker formula, although only 24 of 188 cases were $<37$ weeks GA, and this could have resulted in poorer generalizability of their EFW formula at low gestations. Fetal MRI studies, where fetal fat volumes have been reliably measured in utero, confirm varying fat densities across GAs with negligible fat deposits at around 28 weeks [44]. In the future, MRI may further aid our understanding of developmental fat depositions and lead to more accurate EFW formulae in the setting of FGR or gestational diabetes $[45,46]$.

\section{Role of MRI}

As MRI acquisition speed and affordability improves, MRI will become more important for the preterm fetus, when US quality is limited and an accurate EFW is clinically relevant. Although yet to be fully established in the care pathway, clinical validation of MRI applications must be emphasised, particularly as new MRI-specific fetal growth charts and MRI organ volumes indexed to whole fetal volume are developed $[47,48]$.

While this study provides needed insight into how the MRI-EFW performs at GA remote from term, there are some limitations. The semiautomated MRI segmentation technique used in this study is currently time-consuming (approximately $30 \mathrm{~min} /$ case). Other authors have described bespoke planimetry and manufacturer-based automated segmentations which can further increase reliability and reduce the postprocessing time to $5 \mathrm{~min}$ [49, 43]. Future work should address the development of MRI 3D motion-corrected tools for the fetal body and fully automated techniques for volume extraction. This could include an assessment of methods to address artifacts from reconstructions or segmentation techniques, for example, slice interpolation or 3D smoothing algorithms $[50,51]$.

The primary comparator was US as the gold standard; however, US is subject to well-documented observer subjectivity and measurement error. In the PTB sub-analysis, the MRI-EFW and US-EFW comparator was derived from the ABW; however, charts to calculate birthweight centiles are based on different populations to the fetal growth charts and also takes into account fetal sex [52, 53]. Weight at birth is likely to be physiologically larger to weight in utero due to the mode of delivery, whereby cesarean newborns typically weigh more than standard vaginal deliveries, and the use of intravenous fluids during labor has been seen to artificially increase newborn weight as a result of this intervention [54-56]. These factors were not controlled for in this study, although clinically appropriate newborn and fetal growth charts and use of centiles helped to standardize the comparisons.

\section{Conclusion}

The US-EFW remains the preferred method for fetal growth assessment. Clinicians must be aware of the limitations due to the measurement error and potential clinical implications of using the US-EFW to inform patient management. The MRI-EFW has far lower observer variation than that of US, but the current formulae (Baker and Kacem) are not reliable for the midpregnancy or extremely preterm fetus.

\section{Acknowledgments}

We would like to thank the parents for participating in this study. We also thank the radiographers, sonographers, clinicians, researchers, and administrative staff who assisted with this work, specifically acknowledging the sonographer Tara Fletcher (TF).

\section{Statement of Ethics}

The study has been granted NHS R\&D and ethics approval, NRES ref No. 14/LO/1086 and 07/H0707/105, and all participants gave written informed consent.

\section{Conflict of Interest Statement}

The authors have no conflicts of interest to declare.

\section{Funding Sources}

This work was supported by the Wellcome Trust and Engineering and Physical Sciences Research Council IEH Award (102431) for the Intelligent Fetal Imaging and Diagnosis project (www.ifindproject.com). The authors acknowledge financial support from 
Tommy's charity and the Department of Health via the National Institute for Health Research (NIHR) comprehensive Biomedical Research Centre aware to Guy's and St. Thomas' NHS Foundation Trust in partnership with King's College London and King's College Hospital NHS Foundation Trust.

\section{Author Contributions}

All the authors meet the 4 ICMJE criteria for authorship. Specifically, J.M., D.P., and M.R. for the concept and design; J.M. for data analysis and interpretation; J.M., L.S., C.G., C.L.K., and A.D. for data acquisition and analysis; J.M. and E.S. for the major draft; and all the authors for minor revisions and contribution to intellectual content.

\section{Data Availability Statement}

The collected and analysed data sets used during the current study are available from the corresponding author upon request. Publication and distribution of MRI iFIND project data (14/ LO/1086) are currently ongoing.

\section{References}

1 Sharma D, Shastri S, Farahbakhsh N, Sharma P. Intrauterine growth restriction: part 1. J Matern Fetal Neonatal Med. 2016;29:397787.

2 Royal College of Obstetricians \& Gynaecologists. The investigation and management of the small for gestational age fetus. Green-top guidel no 31; 2013.

3 Miranda J, Rodriguez-Lopez M, Triunfo S, Sairanen M, Kouru H, Parra-Saavedra M, et al. Prediction of fetal growth restriction using estimated fetal weight versus a combined screening model in the third trimester. Ultrasound Obstet Gynecol. 2017;50(5):603

4 Milner J, Arezina J. The accuracy of ultrasound estimation of fetal weight in comparison to birth weight: a systematic review. Ultrasound. 2018;26:32-41.

5 Zhang-Rutledge K, Mack LM, Mastrobattista JM, Gandhi M. Significance and outcomes of fetal growth restriction below the 5 th percentile compared to the 5 th to 10 th percentiles on midgestation growth ultrasonography. J Ultrasound Med. 2017;37(9):2273.

6 Kehl S, Schmidt U, Spaich S, Schild RL, Sütterlin M, Siemer J. What are the limits of accuracy in fetal weight estimation with conventional biometry in 2-dimensional ultrasound? A novel postpartum study. Ultrasound Obstet Gynecol. 2012;39(5):543.

7 Chauhan SP, Cole J, Sanderson M, Scarda JA. Suspicion of intrauterine growth restriction: use of abdominal circumference alone or estimated fetal weight below 10\%. J Matern Neonatal Med. 2006;19(9):557.

8 Dudley NJ. A systematic review of the ultrasound estimation of fetal weight. Ultrasound Obstet Gynecol. 2005;25:80-9.

9 Scioscia M, Vimercati A, Ceci O, Viccino M, Selvagi AE. Estimation of birth weight by twodimensional ultrasonography: a critical appraisal of its accuracy. Obstet Gynecol. 2008; 111(1):58.

10 Kadji C, Camus MF, Bevilacqua E, Cannie MM, Sanchez TC, Jani JC. Repeatability of estimated fetal weight comparison between MR imaging versus 2D ultrasound in at- and nearterm patients. Eur J Radiol. 2017;91:35.
11 Carlin A, Kadji C, De Angelis C, Cannie MM, Jani JC. Prenatal prediction of small-for-gestational age neonates using MR imaging: comparison with conventional 2D ultrasound. J Matern Neonatal Med. 2018;38(10): 1673.

12 Kadji C, Cannie MM, Resta S, Guez D, AbiKhalil F, De Angelis R, et al. Magnetic resonance imaging for prenatal estimation of birthweight in pregnancy: review of available data, techniques, and future perspectives. Am J Obstet Gynecol. 2019 May;220(5):428.

13 Kadji C, Bevilacqua E, Hurtado I, Carlin A, Cannie MM, Jani JC. Comparison of conventional 2D ultrasound to magnetic resonance imaging for prenatal estimation of birthweight in twin pregnancy. Am J Obstet Gynecol. 2018 Jan;218(1):128.e1.

14 Kacem Y, Cannie MM, Kadji C, Dobrescu O, Lo Zito L, Ziane S, et al. Fetal weight estimation: comparison of two-dimensional US and MR imaging assessments. Radiology. 2013 Jun;267(3):902.

15 Zaretsky MV, Reichel TF, McIntire DD, Twickler DM. Comparison of magnetic resonance imaging to ultrasound in the estimation of birth weight at term. Am J Obstet Gynecol. 2003 Oct;189(4):1017.

16 Lo Zito L, Kadji C, Cannie M, Kacem Y, Strizek B, Mbonyumutwa M, et al. Determination of fetal body volume measurement at term with magnetic resonance imaging: effect of various factors. J Matern Neonatal Med. 2013 Aug;26(12):1254.

17 Malin GL, Bugg GJ, Takwoingi Y, Thornton JG, Jones NW. Antenatal magnetic resonance imaging versus ultrasound for predicting neonatal macrosomia: a systematic review and meta-analysis. BJOG. 2016 Jan;123(1):77.

18 Hassibi S, Farhataziz N, Zaretsk M, McIntire D, Twickler DM. Optimization of fetal weight estimates using MRI: comparison of acquisitions. Am J Roentgenol. 2004 Aug;183(2):487.

19 Baker PN, Johnson IR, Gowland PA, Hykin J, Harvey PR, Freeman A, et al. Fetal weight estimation by echo-planar magnetic resonance imaging. Lancet. 1994 Mar 12; 343(8898):644.
20 Lima JC, Miyague AH, Filho FM, Nastri CO, Martins WP. Biometry and fetal weight estimation by two-dimensional and three-dimensional ultrasonography: an intraobserver and interobserver reliability and agreement study. Ultrasound Obstet Gynecol. 2012 Aug; 40(2): 186.

21 Bennini JR, Marussi EF, Barini R, Faro C, Peralta CF. Birth-weight prediction by two- and three-dimensional ultrasound imaging. U1trasound Obstet Gynecol. 2010 Apr;35(4): 426.

22 Mohsen LA, Amin MF. 3D and 2D ultrasound-based fetal weight estimation: a single center experience. J Matern Neonatal Med. 2017 Apr;30(7):818.

23 Story L, Zhang T, Steinweg JK, Hutter J, Matthew J, Dassios T, et al. Foetal lung volumes in pregnant women who deliver very preterm: a pilot study. Pediatr Res. 2020;87:1066-71.

24 Kuhrt K, Smout E, Hezelgrave N, Seed PT, Carter J, Shennan AH. Development and validation of a tool incorporating cervical length and quantitative fetal fibronectin to predict spontaneous preterm birth in asymptomatic high-risk women. Ultrasound Obstet Gynecol. 2016;47:104-9.

25 Hezelgrave NL, Shennan AH. Quantitative fetal fibronectin to predict spontaneous preterm birth: a review. Womens Health. 2016; 12:121-8

26 NHS Screening Programmes. Fetal anomaly screening: programme handbook: NHS Screening Programmes; 2015.

27 Matthew J, Malamateniou C, Knight CL, Baruteau KP, Fletcher T, Davidson A, et al. A comparison of ultrasound with magnetic resonance imaging in the assessment of fetal biometry and weight in the second trimester of pregnancy: an observer agreement and variability study. Ultrasound. 2018;26:229-44.

28 Hadlock FP, Harrist RB, Sharman RS, Deter RL, Park SK. Estimation of fetal weight with the use of head, body, and femur measurements: a prospective study. Am J Obstet Gynecol. 1985 Feb 1;151(3):333. 
29 Kottner J, Audige L, Brorson S, Donner A, Gajewski BJ, Hróbjartsson A, et al. Guidelines for reporting reliability and agreement studies (GRRAS) were proposed. Int J Nurs Stud. 2011;48:661-71.

30 Bland JM, Altman DG. Applying the right statistics: analyses of measurement studies. Ultrasound Obstet Gynecol. 2003;22:85-93.

31 Bartlett JW, Frost C. Reliability, repeatability and reproducibility: analysis of measurement errors in continuous variables. Ultrasound Obstet Gynecol. 2008 Apr;31(4):466.

32 Martins WP, Nastri CO. Interpreting reproducibility results for ultrasound measurements. Ultrasound Obstet Gynecol. 2014 Apr; 43(4):479.

33 Coelho Neto MA, Roncato P, Nastri CO, Martins WP. True reproducibility of ultra sound techniques (TRUST): systematic review of reliability studies in obstetrics and gynecology. Ultrasound Obstet Gynecol. 2015; 46:14-20.

34 Bernard R. Fundamentals of Biostatistics. 5th ed. Boston: PWS Publishers; 2011.

35 Royal College of Paediatrics and Child Health R. UK-WHO growth charts: neonatal and infant close monitoring (NICM): $\mathrm{RCPCH}$; 2020. Available from: https://www.rcpch. ac.uk/resources/uk-who-growth-charts-neonatal-infant-close-monitoring-nicm.

36 Intergrowth-21st. INTERGROWTH-21st applications and calculators; 2020. Available from: https://intergrowth21.tghn.org/intergrowth-21st-applications/.

37 Amini H, Wikström J, Ahlström H, Axelsson $\mathrm{O}$. Second trimester fetal magnetic resonance imaging improves diagnosis of non-central nervous system anomalies. Acta Obstet Gynecol Scand. 2011;90:380-9.

38 Sarris I, Ioannou C, Chamberlain P, Ohuma E, Roseman F, Hoch L, et al. Intra- and interobserver variability in fetal ultrasound measurements. Ultrasound Obstet Gynecol. 2012 Mar;39(3):266.
39 Sarris I, Ioannou C, Dighe M, Mitidieri A, Oberto M, Qingqing W, et al. Standardization of fetal ultrasound biometry measurements: improving the quality and consistency of measurements. Ultrasound Obstet Gynecol. 2011 Dec;38(6):681.

40 Cavallaro A, Ash ST, Napolitano R, Wanyonyi S, Ohuma EO, Molloholli M, et al. Quality control of ultrasound for fetal biometry: results from the intergrowth-21st project. Ultrasound Obstet Gynecol. 2018 Sep;52(3): 332.

41 Kadji C, Cannie MM, Carlin A, Jani JC. Protocol for the prospective observational clinical study: estimation of fetal weight by MRI to PREdict neonatal MACROsomia (PREMACRO study) and small-for-gestational age neonates. BMJ Open. 2019;9:e027160.

42 Uotila J, Dastidar P, Heinonen T, Ryymin P, Punnonen R, Laasonen E. Magnetic resonance imaging compared to ultrasonography in fetal weight and volume estimation in diabetic and normal pregnancy. Acta Obstet Gynecol Scand. 2000 Apr;79(4):255.

43 Anblagan D, Deshpande R, Jones NW, Costigan C, Bugg G, Raine-Fenning N, et al. Measurement of fetal fat in utero in normal and diabetic pregnancies using magnetic resonance imaging. Ultrasound Obstet Gynecol. 2013;42:335-40.

44 Blondiaux E, Chougar L, Gelot A, Valence S, Audureau E, Ducou le Pointe H, et al. Developmental patterns of fetal fat and corresponding signal on T1-weighted magnetic resonance imaging. Pediatr Radiol. 2018;48:31724.

45 Giza SA, Olmstead C, McCooeye DA, Miller MR, Penava DA, Eastabrook GD, et al. Measuring fetal adipose tissue using 3D water-fat magnetic resonance imaging: a feasibility study. J Matern Fetal Neonatal Med. 2020;33: 831-7.

46 Kyriakopoulou V, Vatansever D, Davidson A, Patkee P, Elkommos S, Chew A, et al. Normative biometry of the fetal brain using magnetic resonance imaging. Brain Struct Funct. 2017;222:2295-307.
47 Damodaram MS, Story L, Eixarch E, Patkee P, Patel A, Kumar S, et al. Foetal volumetry using magnetic resonance imaging in intrauterine growth restriction. Early Hum Dev. 2012; 88(Suppl 1):S35-40.

48 Kadji C, De Groof M, Camus MF, De Angelis R, Fellas S, Klass M, et al. The use of a software-assisted method to estimate fetal weight at and near term using magnetic resonance imaging. Fetal Diagn Ther. 2017;41:307-13.

49 Alansary A, Rajchl M, McDonagh SG, Murgasova M, Damodaram M, Lloyd F, et al. PVR: patch-to-volume reconstruction for large area motion correction of fetal MRI. IEEE Trans Med Imaging. 2017.

50 Uus A, Zhang T, Jackson LH, Roberts TA, Rutherford MA, Hajnal JV, et al. Deformable slice-to-volume registration for motion correction of fetal body and placenta MRI. IEEE Trans Med Imaging. 2020;39:2750-9.

51 Stirnemann J, Villar J, Salomon LJ, Ohuma E Ruyan P, Altman DG, et al. International estimated fetal weight standards of the intergrowth-21st project. Ultrasound Obstet Gynecol. 2017 Apr;49(4):478.

52 Wright CM, Williams AF, Elliman D, Bedford $\mathrm{H}$, Birks E, Butler G, et al. Using the new UKWHO growth charts. BMJ. 2010;340:c114050.

53 Elter K, Ay E, Erenus M. Does birthweight affect the mode of delivery? Eur J Obstet Gynecol Reprod Biol. 2003;109(2):138-40.

54 Noel-Weiss J, Woodend AK, Peterson WE, Gibb W, Groll DL. An observational study of associations among maternal fluids during parturition, neonatal output, and breastfed newborn weight loss. Int Breastfeed J. 2011;6: 9.

55 Dawood F, Dowswell T, Quenby S. Intravenous fluids for reducing the duration of labour in low risk nulliparous women. Cochrane Database Syst Rev. 2013.

56 Carlin A, Kadji C, Cannie MM, Resta S, Kang $\mathrm{X}$, Jani JC. The use of magnetic resonance imaging in the prediction of birthweight. Prenat Diagn. 2020 Jan;40(1):125-35. 